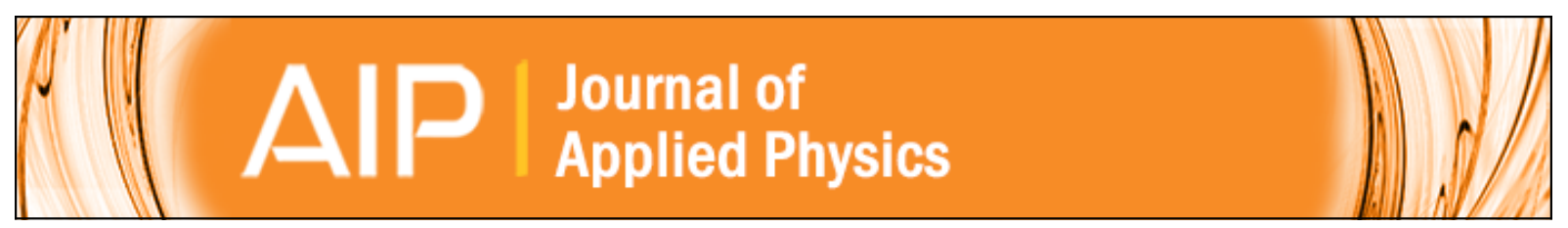

Exchange bias in thin Heusler alloy films in contact with antiferromagnet

J. Dubowik, I. Gociaska, K. Zaski, H. Gowiski, Y. Kudryavtsev, and A. Ehresmann

Citation: Journal of Applied Physics 113, 193907 (2013); doi: 10.1063/1.4807167

View online: http://dx.doi.org/10.1063/1.4807167

View Table of Contents: http://scitation.aip.org/content/aip/journal/jap/113/19?ver=pdfcov

Published by the AIP Publishing

Advertisement:

AlP Re-register for Table of Content Alerts

Create a profile.

Sign up today! 


\title{
Exchange bias in thin Heusler alloy films in contact with antiferromagnet
}

\author{
J. Dubowik, ${ }^{1, a)}$ I. Gościańska, ${ }^{2}$ K. Załęski, ${ }^{1}$ H. Głowiński, ${ }^{1}$ Y. Kudryavtsev, ${ }^{3}$ \\ and A. Ehresmann ${ }^{4}$ \\ ${ }_{1}^{1}$ Institute of Molecular Physics, Polish Academy of Sciences, M. Smoluchowskiego 17, PL-60179 Poznań, \\ Poland \\ ${ }^{2}$ Faculty of Physics, A. Mickiewicz University, Umultowska 85, PL-61614 Poznań, Poland \\ ${ }^{3}$ Institute of Metal Physics, National Academy of Sciences of Ukraine, Vernadsky Ave. 36, Kiev-142, Ukraine \\ ${ }^{4}$ Institute of Physics and Center for Interdisciplinary Nanostructure Science and Technology, \\ University of Kassel, H.Plett Str. 40, D-34132 Kassel, Germany
}

(Received 4 March 2013; accepted 3 May 2013; published online 21 May 2013)

\begin{abstract}
Exchange bias is studied in thin film systems, in which three types of Heusler alloys $\left(\mathrm{Ni}_{2} \mathrm{MnSn}_{\mathrm{Sn}}\right.$ $\mathrm{Co}_{2} \mathrm{MnSn}$, and $\mathrm{Co}_{2} \mathrm{FeSi}$ ) are in contact with an antiferromagnet. Magnetic exchange interactions between the constituting atoms (i.e., Ni-Mn, Mn-Mn, Co-Mn, and Co-Fe, or Co-Co) differ substantially in these Heusler alloys. We explain the influence of the exchange stiffness $A$ within the Heusler alloys and of the exchange coupling between Heusler alloy and an antiferromagnet in a finite interface volume. Insertion of an ultrathin Co layer at interfaces brings about an enhancement of the exchange bias in Heusler alloy/antiferromagnet layer system. (C) 2013 AIP Publishing LLC. [http://dx.doi.org/10.1063/1.4807167]
\end{abstract}

\section{INTRODUCTION}

Exchange bias (EB) occurs as a result of exchange coupling between magnetic moments of an antiferromagnet (AFM) and a ferromagnet (FM) at the AFM/FM interface and is extensively used to pin one of the FM electrodes in spin-valves (SV) or magnetic tunnel junctions (MTJ). EB refers to a unidirectional shift of the FM hysteresis loop from zero field - the exchange bias field $H_{E B}$. EB is also accompanied by an increase in coercivity $H_{C}$ with respect to the unbiased FM. $H_{E B}$ as a result of exchange coupling depends in a complex way on the microstructural factors of the layer system. ${ }^{1}$

In FM/AFM structures with typical FM like Ni-Fe, Co, $\mathrm{Co}-\mathrm{Fe}$, interatomic exchange interactions within the FM layer are regarded as "dense" - the magnetic moments reside on all atoms and they are all involved in interatomic exchange interactions. In FM $\mathrm{X}_{2} \mathrm{YZ}$ Heusler alloys (HA), the magnetic moments mainly reside on $\mathrm{Y}$ (or $\mathrm{X}$ and $\mathrm{Y}$ ) and exchange interactions are either direct between $\mathrm{X}-\mathrm{Y}$ atoms or are mediated by RKKY interactions between $\mathrm{Y}$ atoms. ${ }^{2,3}$ This makes HA distinct from conventional ferromagnets.

Structural disorder has a great influence on the magnetic properties of HA, and hence on the exchange interactions. ${ }^{4-6}$ Structural disorder in a HA can be reduced by proper annealing $^{4}$ with the aim of achieving the ideal L2 $2_{1}$ structure. ${ }^{7}$ For SV or MTJ with HA, the entire structure is usually deposited at room temperature (RT) and then it is subjected to annealing at $400-500^{\circ} \mathrm{C}$ and field cooling to enhance chemical (site) ordering and setting AFM, respectively. ${ }^{7,8}$ Such a procedure results in a relatively low interface roughness ${ }^{7,9}$ and low interdiffusion. ${ }^{10}$

Some HA are interesting because of their potential to produce a high spin polarization in magnetoresistive

${ }^{a)}$ Electronic mail: dubowik@ifmpan.poznan.pl devices. ${ }^{4}$ Therefore, it is hoped that the use of an HA in contact with an AFM as the pinned reference layer would lead to SV or MTJ with ultrahigh magnetoresistive effect amplitudes. Reports on EB in bilayers containing HA are still rare and are restricted to a few reports on HA/AFM bilayers in MTJs including $\mathrm{Co}_{2} \mathrm{MnSi} / \mathrm{IrMn},{ }^{10,11} \mathrm{Co}_{2} \mathrm{FeAl} / \mathrm{IrMn},{ }^{12}$ or $\mathrm{Co}_{2} \mathrm{FeSi} / \mathrm{IrMn}^{13-15}$ bilayers. In particular, it was shown that $\mathrm{Co}_{2} \mathrm{MnSi} / \mathrm{Al}-\mathrm{O} / \mathrm{Co}_{2} \mathrm{MnSi} / \mathrm{IrMn} \mathrm{MTJ}$ can exhibit a large tunnel magnetoresistance of $570 \%$ at low temperatures. ${ }^{10}$ Ohdaira et al. reported a spin transistor structure consisting of two MTJ with $\mathrm{Co}_{2} \mathrm{MnSi}$ with excellent characteristics. ${ }^{11}$ Endo et al. reported grain size distribution influence of both AFM IrMn and $\mathrm{Co}_{2} \mathrm{FeSi}$ layers on the magnetic properties of exchange-biased films with $H_{E B}$ of $100-2000$ Oe. $^{14,15}$ They showed that sharp interfaces matching is required between 8 and $10 \mathrm{~nm}$ IrMn grains and $15 \mathrm{~nm} \mathrm{Co} \mathrm{Co}_{2} \mathrm{FeSi}$ grains to exhibit a large $H_{E B}$. Optimized annealing led to $H_{E B}$ up to $200 \mathrm{Oe}$ and insertion of a $0.5 \mathrm{~nm}$ Mn layer can increase EB by a factor of two. ${ }^{15}$ Alley et al. showed that insertion of CoFe layer $(0.5-2 \mathrm{~nm})$ results in $15 \%$ enhancement of EB. ${ }^{13}$

In this paper, we report magnetic properties of HA thin films $\left(\mathrm{Ni}_{2} \mathrm{MnSn}, \mathrm{Co}_{2} \mathrm{MnSn}\right.$, and $\left.\mathrm{Co}_{2} \mathrm{FeSi}\right)$ in contact with AFM (NiMn, FeMn, and mainly IrMn). We aim at determining important features that lead to the presence or absence of EB in these structures. We have chosen HA with diverse exchange interatomic interactions, which result in Curie temperatures $\left(T_{C}\right)$ of $1100 \mathrm{~K}, 829 \mathrm{~K}$, and $340 \mathrm{~K}$, for $\mathrm{Co}_{2} \mathrm{FeSi}^{4}{ }^{4}$ $\mathrm{Co}_{2} \mathrm{MnSn},{ }^{4}$ and $\mathrm{Ni}_{2} \mathrm{MnSn},{ }^{2}$ respectively.

\section{EXPERIMENTAL DETAILS}

A series of $\mathrm{HA}\left(\mathrm{Ni}_{2} \mathrm{MnSn}, \mathrm{Co}_{2} \mathrm{MnSn}\right.$, and $\left.\mathrm{Co}_{2} \mathrm{FeSi}\right)$ thin film structures in contact with AFM were deposited onto $\mathrm{Si} /$ $\mathrm{SiO}$ substrates with $\mathrm{Ta}(5-10 \mathrm{~nm})$ as a buffer and a cap layer using an ultra high vacuum magnetron sputtering system. AFM films were deposited from an $\operatorname{Ir}_{17} \mathrm{Mn}_{83}, \mathrm{Fe}_{50} \mathrm{Mn}_{50}$, and $\mathrm{Ni}_{50} \mathrm{Mn}_{50}$ alloy targets. The base pressure was better than 
$4 \times 10^{-8} \mathrm{~Pa}$ and the Ar deposition pressure was $2 \times 10^{-5} \mathrm{~Pa}$. HA films were deposited from either separate $\mathrm{Ni}, \mathrm{Mn}_{50} \mathrm{Sn}_{50}$, and Mn targets, ${ }^{9}$ or from $\mathrm{Co}_{50} \mathrm{Mn}_{25} \mathrm{Sn}_{25}$ and $\mathrm{Co}_{50} \mathrm{Mn}_{25} \mathrm{Si}_{25}$ alloy targets. The samples comprise $\mathrm{Si}$-sub/Ta/HA x/AFM $\mathrm{y} / \mathrm{Ta}$ bilayers, bilayers with an ultrathin Co layer $(0.5-1 \mathrm{~nm}$ thick) inserted at the HA/AFM interface- $\mathrm{Si}$-sub/Ta/HA (x)/Co/AFM (y)/Ta and Si-sub/Ta/[AFM (y)/HA (x)] $\times$ N/AFM (y)/Ta or Si-sub/Ta/[AFM (y)/Co/HA (x)/Co] $\times$ N/AFM (y)/Ta multilayers. The numbers (and $x, y$ ) denote thicknesses in nanometers and $\mathrm{N}$ is the number of repetitions, which was 3 or 5. The thicknesses $x$ and $y$ were in range of $x=5-15 \mathrm{~nm}$ and $y=10-30 \mathrm{~nm}$ for the HA and AFM, respectively, and were approximately the same as that reported in Ref. 15 . Structural characterization was carried out by X-ray diffraction (XRD) (see Ref. 9, for details) and transmission electron microscopy (TEM). Chemical analysis of HA and AFM was performed with energy-dispersive X-ray fluorescence and confirmed appropriate stoichiometry of the films within $\pm 3 \%$. Magnetic measurements were made on a home-made vibration sample magnetometer (VSM) calibrated with a Co standard film. Temperature measurements were made by a Physical Property Measurement System (Quantum Design) from 5 to $300 \mathrm{~K}$. The samples were usually deposited at RT to have smooth interfaces and then they were annealed at $400{ }^{\circ} \mathrm{C}$ for $15-30 \mathrm{~min}$ to transform the HA into the desired $\mathrm{L} 22_{1}$ phase and then finally cooled down to set in the AFM in a field of $1 \mathrm{kOe}$ for exchange bias initialization.

The multilayer stack quality was characterized by interface roughness, interface volume, and density profiles monitored by X-ray reflectivity (XRR) spectra. Figures 1 and 2 show XRR of a typical $\mathrm{Ni}_{2} \mathrm{MnSn} / \mathrm{IrMn}$ bilayer and $\mathrm{a} \mathrm{Co}_{2} \mathrm{FeSi} /$ $\mathrm{Co} / \mathrm{IrMn}$ multilayer, respectively. The lines show the fits according to a model structures known from the deposition procedure performed by a fitting using a SIMULREFLECT software ${ }^{16}$ under the assumption that the density profiles are rectangular with RMS roughnesses in the range of $0.5-1 \mathrm{~nm}$. For

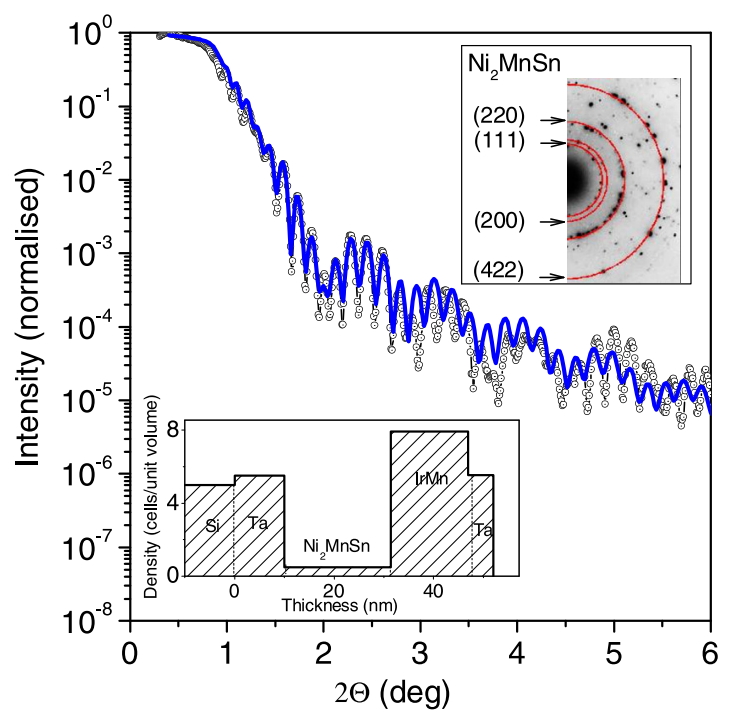

FIG. 1. X-ray reflectivity versus grazing angle of a $\mathrm{Si} / \mathrm{Ta}(10) / \mathrm{Ni}_{2} \mathrm{MnSn}$ (20)/IrMn (15)/Ta (5) thin film structure (circles) and a fit (full line) with a density profile (expressed as number of the unit cells per unit volume) of individual layers shown in the bottom inset. The upper inset shows electron diffraction pattern of a single $\mathrm{Ni}_{2} \mathrm{MnSn}$ film.

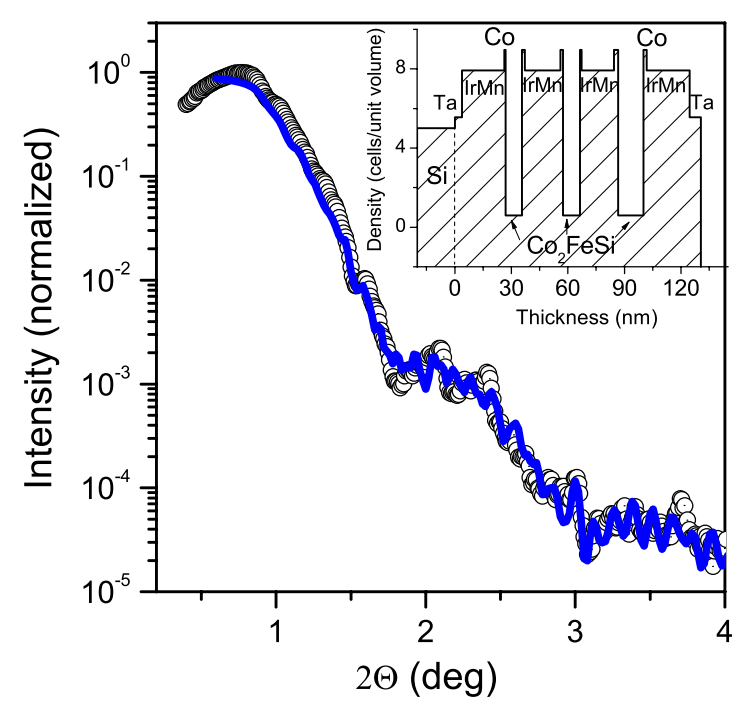

FIG. 2. X-ray reflectivity versus grazing angle of a Si/Ta (5)/[IrMn (20)/Co (1) $\left./ \mathrm{Co}_{2} \mathrm{FeSi}(10)\right] \times 3 / \mathrm{IrMn}(20) / \mathrm{Ta}$ (5) thin film structure (circles) and a fit (full line) with a density profile of individual layers shown in the inset.

example, the fitting procedure of XRR data shown in Fig. 1 gives $0.35,0.5,0.3$, and $1.2 \mathrm{~nm}$ RMS roughness for Ta, $\mathrm{Ni}_{2} \mathrm{MnSn}$, IrMn, and Ta layers, respectively. The density of an individual layer is defined as number of the unit cells per unit volume. ${ }^{16}$ The density profiles of the layers are shown in Figs. 1 and 2 as the insets. The general characteristics of the XRR are well fitted despite the simplicity of the fitting model. XRR analysis suggests that our technology (i.e., sputtering deposition at RT, post-deposition annealing, and field cooling to set AFM) ensures the HA/AFM structures with composition profiles similar to the nominal ones and with moderate interface roughnesses of $0.5-1 \mathrm{~nm}$. The upper right inset in Fig. 1 shows TEM diffraction of a typical $\mathrm{Ni}_{2} \mathrm{MnSn}$ film. The presence of (111) and (200) superstructure reflections suggests that the film indeed possesses B2 or $\mathrm{L} 2$ ordering typical for a $\mathrm{HA}_{1}{ }^{4}$

\section{RESULTS}

In the as-deposited state, both $\mathrm{Ni}_{2} \mathrm{MnSn}$ and $\mathrm{Co}_{2} \mathrm{MnSn}$ are nonmagnetic at $\mathrm{RT}$. In contrast, $\mathrm{Co}_{2} \mathrm{FeSi}$ is already ferromagnetic in a disordered state with a saturation magnetization $M_{S}$ of $700 \mathrm{G}$. Therefore, a proper annealing of the layers is important both for ordering of HA layers and for setting AFM layers. After annealing at $400^{\circ} \mathrm{C}$ for $15-30 \mathrm{~min}$, magnetization measurements at $5 \mathrm{~K}$ yield $M_{S}$ of $690 \mathrm{G}\left(4 \mu_{B} /\right.$ f.u. $)$, $820 \mathrm{G}\left(4.8 \mu_{B} /\right.$ f.u. $)$, and $1000 \mathrm{G}\left(5 \mu_{B} /\right.$ f.u. $)$ for $\mathrm{Ni}_{2} \mathrm{MnSn}$, $\mathrm{Co}_{2} \mathrm{MnSn}$, and $\mathrm{Co}_{2} \mathrm{FeSi}$ films, respectively. We estimate the error in determination of the magnetization to about $10 \%$. The uncertainty about magnetization originates mainly from error in the estimation of the sample volume. The values in parentheses are expressed in the numbers of Bohr magnetons per formula unit (f.u.). The maximum $M_{S}$ value for $\mathrm{Co}_{2} \mathrm{FeSi}$ after annealing is $92 \%$ of that reported for epitaxially grown thin film of $5.1 \mu_{B} /$ f.u. ${ }^{17}$ The values of $M_{S}$ can be compared with those presented in Table I.

No EB was obtained at RT in the structures comprising $\mathrm{Ni}_{2} \mathrm{MnSn} / \mathrm{NiMn}, \mathrm{Ni}_{2} \mathrm{MnSn} / \mathrm{FeMn}$, or $\mathrm{Ni}_{2} \mathrm{MnSn} / \mathrm{IrMn}$ despite their good structural order (Fig. 1). However, if the hysteresis loops are measured with the magnetic field applied 
TABLE I. Magnetic properties of $\mathrm{X}_{2} \mathrm{YZ}$ Heusler alloys: $m$ is the calculated magnetic moment per f.u. in $\mu_{B} ; m_{X}$ and $m_{Y}$ are the magnetic moments on $\mathrm{X}$ and $\mathrm{Y}$ site, respectively; $J_{X-X}, J_{X-Y}$, and $J_{Y-Y}$ are the exchange integrals between $X-X, X-Y$, and $Y-Y$ nearest neighbors, respectively; $A$ is the exchange constant; $T_{C}$ is the Curie temperature.

\begin{tabular}{|c|c|c|c|c|c|c|c|c|}
\hline $\begin{array}{l}\text { Heusler } \\
\text { alloy }\end{array}$ & $\begin{array}{c}m \\
\mu_{B} / \text { f.u. }\end{array}$ & $\begin{array}{c}m_{X} \\
\mu_{B}\end{array}$ & $\begin{array}{l}m_{Y} \\
\mu_{B}\end{array}$ & $\begin{array}{c}J_{X-X}{ }^{\mathrm{a}} \\
\mathrm{mRy}\end{array}$ & $\begin{array}{c}J_{X-Y^{\mathrm{a}}} \\
\mathrm{mRy}\end{array}$ & $\begin{array}{c}J_{Y-Y^{\mathrm{a}}} \\
\mathrm{mRy}\end{array}$ & $\begin{array}{c}A \times 10^{6} \\
\mathrm{erg} / \mathrm{cm}\end{array}$ & $\begin{array}{l}T_{C} \\
\mathrm{~K}\end{array}$ \\
\hline $\mathrm{Ni}_{2} \mathrm{MnSn}$ & 4.0 (Ref. 28) & 0.14 (Ref. 28) & 3.50 (Ref. 28) & $\approx 0$ & 0.2 & 0.2 & 0.1 (Ref. 9) & 340 (Ref. 2) \\
\hline $\mathrm{Co}_{2} \mathrm{MnSn}$ & 5.0 (Ref. 21) & 0.97 (Ref. 21) & 3.23 (Ref. 21) & 0.1 & 0.8 & 0.1 & 0.6 (Ref. 22) & 829 (Ref. 4) \\
\hline $\mathrm{Co}_{2} \mathrm{FeSi}$ & 6.0 (Ref. 21) & $1.40($ Ref. 21$)$ & 2.87 (Ref. 21) & 0.4 & 1.5 & 0.05 & 3.2 (Ref. 22) & 1100 (Ref. 4) \\
\hline Permalloy & $\approx 4$ (Ref. 1) & $\ldots$ & $\ldots$ & $\ldots$ & 0.3 & $\ldots$ & $1.0($ Ref. 1$)$ & 800 (Ref. 1) \\
\hline
\end{tabular}

${ }^{\mathrm{a}}$ The values of the exchange integrals are calculated by the EMTO package. ${ }^{27}$

parallel to the anisotropy axis imposed by field cooling from high temperatures, some EB is observed at low temperatures. As it is shown in Fig. 3, EB in the $\mathrm{Ni}_{2} \mathrm{MnSn} / \mathrm{NiMn}$ bilayer is observed at temperatures below $50 \mathrm{~K}$ and is accompanied by a very high coercive field $H_{C}$ of $500 \mathrm{Oe}$, which decreases to 60 Oe at $300 \mathrm{~K}$. The inset of Fig. 3 shows the hysteresis loop of a single $\mathrm{Ni}_{2} \mathrm{MnSn}$ film $(\approx 15 \mathrm{Oe}$ at $\mathrm{RT})$ in comparison to that of the $\mathrm{Ni}_{2} \mathrm{MnSn} / \mathrm{NiMn}$ bilayer at $5 \mathrm{~K}$. Similarly to lowtemperature EB observed in off-stoichiometric Ni-Mn-Sn bulk alloys ${ }^{18}$ and various types of Ni-Mn-Sn thin film structures, ${ }^{19}$ the $\mathrm{EB}$ in $\mathrm{Ni}_{2} \mathrm{MnSn} / \mathrm{NiMn}$ bilayer can probably be ascribed to the presence of local FM/AFM interactions in nanoscale. Westerholt et al. ${ }^{20}$ have suggested that the lowtemperature $\mathrm{EB}$ in multilayers containing $\mathrm{HA}$ originates from a spin glass type of magnetic order for a thin interlayer at the interfaces. We will show later in Sec. III how to distinguish the latter EB from that due FM/AFM exchange coupling present at RT.

Figure 4 compares typical RT hysteresis loops of HA/ AFM bilayers (continuous lines) with those of single HA films of the same thickness and of low coercivity of several Oe (dotted lines). For $\mathrm{Ni}_{2} \mathrm{MnSn} / \mathrm{IrMn}$ bilayer (Fig. 4(a)), the only

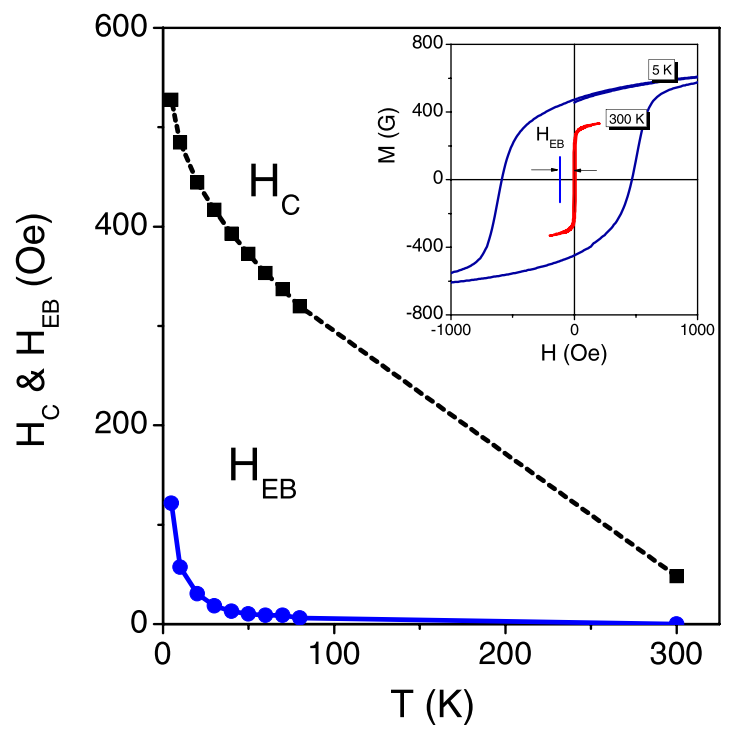

FIG. 3. Temperature dependence of coercivity $H_{C}$ and exchange bias field $H_{E B}$ for a $\mathrm{Ni}_{2} \mathrm{MnSn}(30) / \mathrm{NiMn}(50)$ bilayer measured with the magnetic field applied parallel to the anisotropy axis imposed by field cooling from high temperatures. Inset shows hysteresis loops for $\mathrm{Ni}_{2} \mathrm{MnSn}$ (30)/ $\mathrm{NiMn}(50)$ bilayer at $5 \mathrm{~K}$ (blue) and for a single $30 \mathrm{~nm} \mathrm{Ni}_{2} \mathrm{MnSn}$ film at $300 \mathrm{~K}$ (red). The numbers in parentheses denote thickness in $\mathrm{nm}$. effect is a considerable increase in coercivity $\left(H_{C}=56 \mathrm{Oe}\right)$ in comparison to $H_{C}=15 \mathrm{Oe}$ for a single $\mathrm{Ni}_{2} \mathrm{MnSn}$ film. As it is shown in Figs. 4(b)-4(d), hysteresis loops are already shifted with $H_{E B}$ of 3, 14, and $50 \mathrm{Oe}$ for $\mathrm{Co}_{2} \mathrm{MnSn} / \mathrm{FeMn}, \mathrm{Co}_{2} \mathrm{MnSn} /$ IrMn, and $\mathrm{Co}_{2} \mathrm{FeSi} / \mathrm{IrMn}$ bilayers, respectively. The coercivity of the FM/AFM bilayers shown in Fig. 4 increases due to an enhancement of exchange coupling from $56 \mathrm{Oe}$ for $\mathrm{Ni}_{2} \mathrm{MnSn} /$ IrMn with no EB to $230 \mathrm{Oe}$ for $\mathrm{Co}_{2} \mathrm{FeSi} / \mathrm{IrMn}$ with the highest EB. Nevertheless, as it is shown in Fig. $4, H_{E B}$ in our HA/ AFM bilayers is low and merely amounts to $0 \%, 3 \%, 7 \%$, and $22 \%$ of $H_{C}$ for $\mathrm{Ni}_{2} \mathrm{MnSn} / \mathrm{IrMn}, \mathrm{Co}_{2} \mathrm{MnSn} / \mathrm{FeMn}, \mathrm{Co}_{2} \mathrm{MnSn} /$ $\mathrm{IrMn}$, and $\mathrm{Co}_{2} \mathrm{FeSi} / \mathrm{IrMn}$, respectively. Moreover, in opposite to results of Ref. 15, EB in our bilayer $\mathrm{Co}_{2} \mathrm{MnSn} / \mathrm{IrMn}$ and $\mathrm{Co}_{2} \mathrm{FeSi} / \mathrm{IrMn}$ structures significantly depends on annealing time. We have not done systematic studies on the annealing time dependence of the exchange bias in our HA/AFM systems. However, we checked that an annealing at $300^{\circ} \mathrm{C}$ did

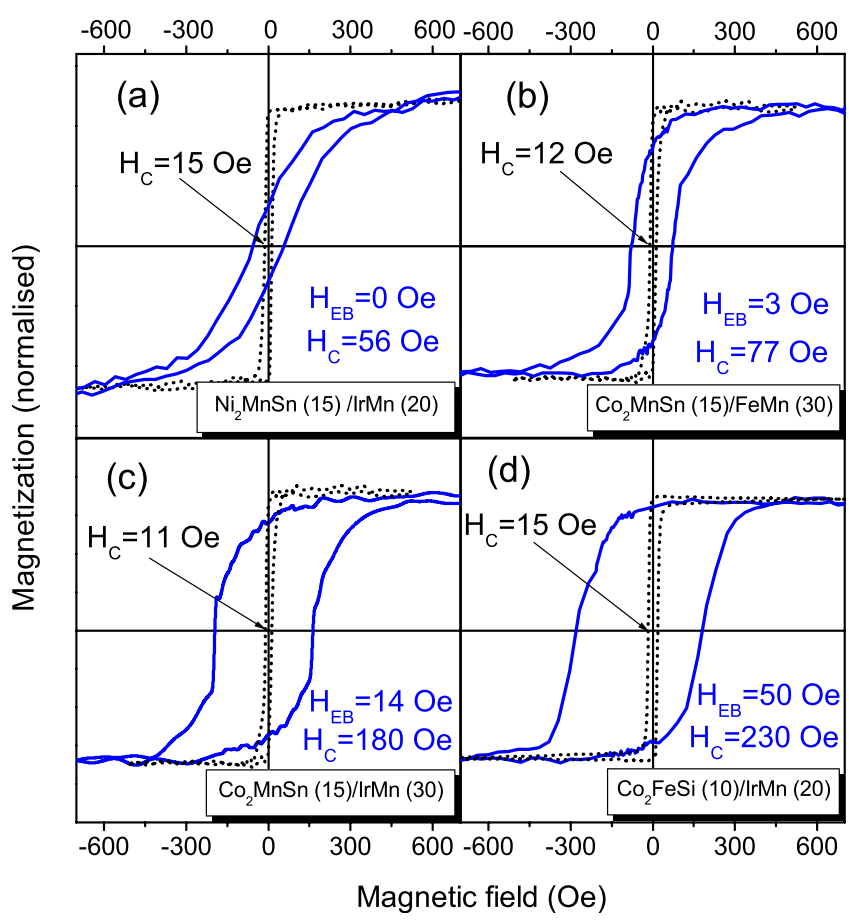

FIG. 4. Room temperature hysteresis loops (dotted lines, black) of single HA films with small coercivity $H_{C}$ values (in black): $\mathrm{Ni}_{2} \mathrm{MnSn}$ (a), $\mathrm{Co}_{2} \mathrm{MnSn}$ (b) and (c), $\mathrm{Co}_{2} \mathrm{FeSi}$ (d) and of HA/AFM bilayers (continuous lines): $\mathrm{Ni}_{2} \mathrm{MnSn}$ (20)/IrMn (15) (a), $\mathrm{Co}_{2} \mathrm{MnSn}$ (15)/FeMn (30) (b), $\mathrm{Co}_{2} \mathrm{MnSn}(15) / \mathrm{IrMn}$ (30) (c), $\mathrm{Co}_{2} \mathrm{FeSi}$ (10)/IrMn (20) (d). The values of $H_{E B}$ and $H_{C}$ (in blu) relate to exchange-biased bilayers. The numbers in parentheses denote thickness in $\mathrm{nm}$. 
not lead to a high ordering of HA. On the other hand, the annealing at $450{ }^{\circ} \mathrm{C}$ resulted in interdiffusion of the structures with HA layers $5 \mathrm{~nm}$ thick. Therefore, basing on Refs. 10 and 13 , we chose annealing at $400^{\circ} \mathrm{C}$ for $15 \mathrm{~min}$ as optimal to obtain a sufficient ordering of HA with no substantial interdiffusion. For example, a $\mathrm{Co}_{2} \mathrm{FeSi}(10) / \mathrm{IrMn}$ (20) bilayer reveals $H_{E B}=50 \mathrm{Oe}\left(H_{C}=230 \mathrm{Oe}\right)$ and $H_{E B}=73 \mathrm{Oe}\left(H_{C}=180 \mathrm{Oe}\right)$ after annealing at $400^{\circ} \mathrm{C} / 15 \mathrm{~min}$ and $400^{\circ} \mathrm{C} / 30 \mathrm{~min}$, respectively. This points out on a significant role of IrMn grain size, which has been prepared in Ref. 15 by using a HiTUS sputtering system allowing control of the grain size.

In accordance with Ref. 13, an ultrathin Co layer added at the HA/AFM interface induces an enhancement of EB. As it is seen in Figs. 5(a) and 5(b), a $1 \mathrm{~nm}$ thick Co layer "dusted" at $\mathrm{Co}_{2} \mathrm{MnSn} / \mathrm{FeMn}$ and $\mathrm{Co}_{2} \mathrm{MnSn} / \mathrm{IrMn}$ leads to a slight increase in $H_{E B}$ up to several Oe. On the other hand, a $1 \mathrm{~nm}$ thick Co layer inserted at $\mathrm{Co}_{2} \mathrm{FeSi}(10) / \mathrm{IrMn}(20)$ interface [Fig. 5(c)] results in nearly twofold increase of $H_{E B}$ in comparison with that of $\mathrm{Co}_{2} \mathrm{FeSi}(10) / \mathrm{IrMn}$ (20) shown in Fig. 4(d).

By multiplying the number of HA/AFM interfaces, we aimed at further enhancement of EB in our HA/AFM structures. Additionally, in a few cases we prepared HA/AFM multilayers with $0.5-1 \mathrm{~nm}$ Co added at the interfaces. The main results are given in Fig. 6, in which the RT hysteresis loops of HA/AFM (dashed lines) and HA/Co (0.5-1)/AFM (continuous lines) are shown for comparison. Fig. 6(a) shows that there is no enhancement in EB for [IrMn $(20) / \mathrm{Ni}_{2} \mathrm{MnSn}$ (10) $] \times 5 / \operatorname{IrMn}(20)$ multilayer and $H_{C}$ is still relatively low of $40 \mathrm{Oe}$. The magnetization saturates at a field of $500 \mathrm{Oe}$ as if the $\mathrm{Ni}_{2} \mathrm{MnSn}$ layers possess uniaxial anisotropy with a high dispersion of easy axes. As it is shown in Fig. 6(b), the effect of multiplication of the number of HA/AFM interfaces is more pronounced in multilayers containing $\mathrm{Co}_{2} \mathrm{MnSn}$ with $H_{E B}\left(H_{C}\right)$ of $61 \mathrm{Oe}(220 \mathrm{Oe})$ and $89 \mathrm{Oe}(260 \mathrm{Oe})$ for [IrMn

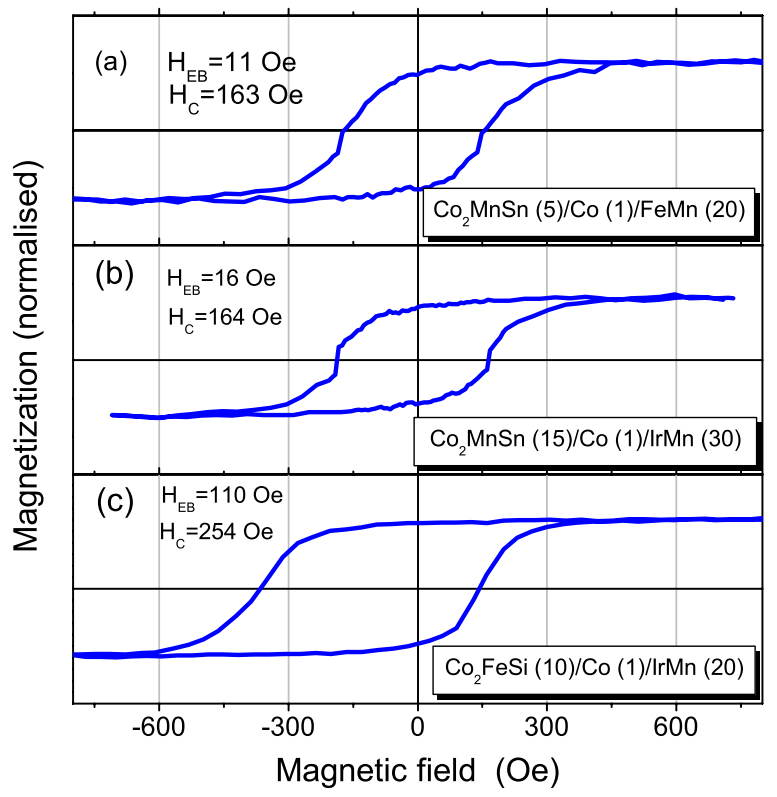

FIG. 5. Effect of ultrathin Co $(1 \mathrm{~nm})$ insertion at interfaces of $\mathrm{X}_{2} \mathrm{YZ}$ HA in contact with FeMn or IrMn: (a) $\mathrm{Co}_{2} \mathrm{MnSn}$ (5)/Co (1)/FeMn (20), (b) $\mathrm{Co}_{2} \mathrm{MnSn}(15) / \mathrm{Co}(1) / \mathrm{IrMn}$ (30), (c) $\mathrm{Co}_{2} \mathrm{FeSi}$ (10)/Co (1)/IrMn (20). The numbers in parentheses denote thickness in $\mathrm{nm}$.

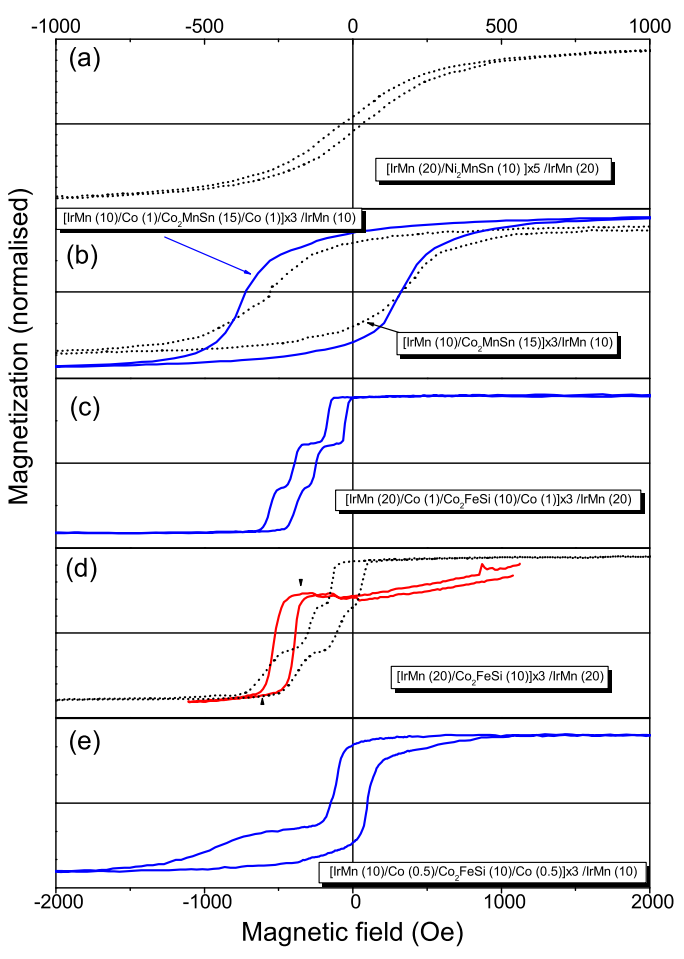

FIG. 6. Typical hysteresis loops of $\mathrm{X}_{2} \mathrm{YZ} / \mathrm{IrMn}$ multilayers without (dashed lines) or with (continuous lines) ultrathin Co layer inserted at the interfaces: (a) $\left[\operatorname{IrMn}(20) / \mathrm{Ni}_{2} \mathrm{MnSn}(10)\right] \times 5 / \operatorname{IrMn}(20)$, (b) $\left[\operatorname{IrMn}(10) / \mathrm{Co}_{2} \mathrm{MnSn}(15)\right]$ $\times 3 / \operatorname{IrMn}(10)$ and $\left[\operatorname{IrMn}(20) / \mathrm{Co}(1) / \mathrm{Co}_{2} \mathrm{MnSn}(15) / \mathrm{Co}(1)\right] \times 3 / \mathrm{IrMn}(20)$, (c) $\left[\operatorname{IrMn}(20) / \mathrm{Co}(1) / \mathrm{Co}_{2} \mathrm{FeSi}(10) / \mathrm{Co}(1)\right] \times 3 / \operatorname{IrMn}(20)$, (d) $[\operatorname{IrMn}(20) /$ $\left.\mathrm{Co}_{2} \mathrm{FeSi}(10)\right] \times 3 / \mathrm{IrMn}(20)$ measured with MOKE (red line) and VSM (dashed line), (e) [ $\left.\mathrm{IrMn}(10) / \mathrm{Co}(0.5) / \mathrm{Co}_{2} \mathrm{FeSi}(10) / \mathrm{Co}(0.5)\right] \times 3 / \mathrm{IrMn}(10)$. The numbers in parentheses denote thickness in $\mathrm{nm}$. The scale of magnetic field is the same for all plots except that in (e).

$\left.(10) / \mathrm{Co}_{2} \mathrm{MnSn}(15)\right] \times 3 / \mathrm{IrMn}(10)$ (dashed lines) and $[\mathrm{IrMn}$ (20)/Co (1)/Co $\left.{ }_{2} \mathrm{MnSn}(15) / \mathrm{Co}(1)\right] \times 3 / \mathrm{IrMn}(20)$ (continuous lines) multilayers, respectively. It is worth noticing that hysteresis loops of $\left[\mathrm{IrMn} / \mathrm{Co} / \mathrm{Co}_{2} \mathrm{MnSn}\right]$ have nearly the same shape as those of bilayers. In opposite, for [ $\mathrm{IrMn} /$ $\left.\mathrm{Co}_{2} \mathrm{FeSi}\right]$ and $\left[\mathrm{IrMn} / \mathrm{Co} / \mathrm{Co}_{2} \mathrm{FeSi}\right]$ multilayers, a "fine" structure of hysteresis loops is frequently observed. As it is seen in Figs. 6(c) and 6(d), $H_{E B}$ increases with the growth in the repetition number as if the bottom stack polarizes the upper one. Using a magneto-optical Kerr (MOKE) magnetometer, which is surface sensitive with penetration depth of $30-40 \mathrm{~nm}$, we checked that the upper $\mathrm{IrMn} / \mathrm{Co}_{2} \mathrm{FeSi} / \mathrm{IrMn}$ stack has the highest EB.

Figure 6(d) clearly shows that the MOKE hysteresis loop (continuous red line) has the highest $H_{E B}$ of $250 \mathrm{Oe}$, which is the same as for the left part of the VSM loop (dashed line). The increase in the Kerr signal under the positive field application is caused by a drift in Kerr rotation due to some influence of the magnetic field on optical components. The value of $250 \mathrm{Oe}$ is in accordance with that reported in Ref. 15 for an $\mathrm{IrMn} / \mathrm{Co}_{2} \mathrm{FeSi}$ bilayer after optimized annealing. Ultrathin Co layers added at $\mathrm{IrMn} / \mathrm{Co}_{2} \mathrm{FeSi}$ interfaces result in a slight enhancement of EB of individual stacks and make the "fine" structures in hysteresis loops even sharper as it is shown in Fig. 6(c). By a slight change in [IrMn (10)/Co (0.5)/ $\left.\mathrm{Co}_{2} \mathrm{FeSi}(10) / \mathrm{Co}(0.5)\right] \times 3 / \mathrm{IrMn}(10)$ geometry, we could still enhance $H_{E B}$ up to about $500 \mathrm{Oe}$ 
[as it is seen in the lower left part of hysteresis loop in Fig. 6(e)] but the "fine" structure of the hysteresis loop in this case is not so sharp. We believe that the upper stack has the highest EB but we cannot confirm it using MOKE due to limitation in magnetic field. Using the Scherrer formula for the Bragg reflections of small particles, we derived that in as-deposited $\mathrm{Co}_{2} \mathrm{FeSi}$ and $\mathrm{IrMn}$ layers the mean size of grains is of $8 \mathrm{~nm}$. After annealing at $400{ }^{\circ} \mathrm{C}$, the respective grain size is of $10-11$ and $8 \mathrm{~nm}$, respectively. It is worth noting, however, that after annealing the intensity of the (111) reflection increased in comparison to that of the (200) reflection. This suggests that the preferred (111) texture of IrMn grains increases after annealing.

Usually, $H_{E B}$ in conventional FM/AFM structures shows only a slight temperature dependence except a temperature region next to blocking temperature. ${ }^{26}$ In most of our HA/ AFM structures, however, a relatively strong increase in $H_{E B}$ is observed at low temperatures (see Ref. 19). We have tentatively connected EB in Ni-Mn-Sn thin film structures with the presence of distributed FM and AFM couplings due to a high heterogeneity in nanoscale in the bulk of the films. ${ }^{19}$ To distinguish the latter effect from EB due to FM/AFM interactions at HA/AFM interfaces, we chose an [IrMn (10)/ $\left.\mathrm{Co}_{2} \mathrm{MnSn}(15)\right] \times 3 / \mathrm{IrMn}(10)$ multilayer with moderate $H_{E B}$ of 40 Oe at RT (see Fig. 6(b)_dashed line) and we measured magnetization reversals in a broad temperature range of 5-300 K with the magnetic field applied either parallel $(H \|)$ or perpendicular $(H \perp)$ to the direction of the anisotropy axis imposed by field cooling from high temperatures. It is seen in Fig. 7 that $H_{E B}^{\perp}$ vs. $T$ for $H \perp$ is always lower than $H_{E B}^{\|}$vs. $T$ for $H \|$. At low temperature, both dependencies reveal a strong increase in $\mathrm{EB}$ and $H_{C}>H_{E B}$, like in $\mathrm{Ni}_{2} \mathrm{MnSn} / \mathrm{NiMn}$ bilayer (Fig. 3). We relate the strong EB at low temperature region in $\mathrm{Ni}_{2} \mathrm{MnSn} / \mathrm{NiMn}$ (Fig. 3) or $\mathrm{Co}_{2} \mathrm{MnSn} / \mathrm{IrMn}$ (Fig. 7)

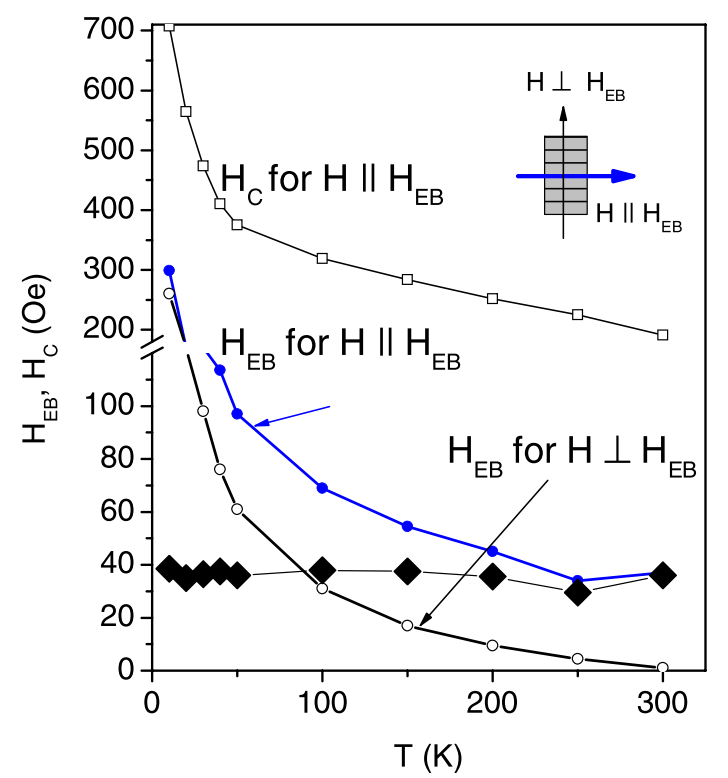

FIG. 7. Temperature dependence of coercivity $H_{C}$ and exchange bias field $H_{E B}$ for [IrMn (10)/ $\left.\mathrm{Co}_{2} \mathrm{MnSn}(15)\right] \times 3 / \mathrm{IrMn}(10)$ multilayer measured with the magnetic field applied parallel $(\|)$ and perpendicular $(\perp)$ to anisotropy axis imposed by field cooling from high temperatures. The diamonds show the temperature dependence of $H_{E B}=H_{E B}^{\|}-H_{E B}^{\perp}$ resulting from FM/AFM interfaces. as resulting from AFM and FM exchange interactions (spinglass like) in the nanoscale in a similar way as EB observed in some bulk HA. ${ }^{33}$ In opposite, as it is shown in Fig. 7 by diamonds, the difference of $H_{E B}^{\|}-H_{E B}^{\perp}$ vs. $T$ is independent of temperature and amounts to $\sim H_{E B}$ (RT). Therefore, we assume that the temperature independent EB is due to pure interface coupling.

\section{DISCUSSION}

We briefly summarize the results concerning EB in our HA/AFM annealed thin film structures as follows. No EB was observed in $\mathrm{Ni}_{2} \mathrm{MnSn} / \mathrm{AFM}$ bilayers and multilayers at RT. The reason might be the low $T_{C}=340 \mathrm{~K}$ of $\mathrm{Ni}_{2} \mathrm{MnSn}$, much less than the Neèl temperature $T_{N}=520 \mathrm{~K}$ (Ref. 24) of IrMn. However, it has been shown that EB can be established in FM/AFM structures regardless of the relative values of $T_{C}$ and $T_{N}{ }^{25}$ Moderate EB (i.e., with $H_{E B}<H_{C}$ ) was observed at $\mathrm{RT}$ in $\mathrm{Co}_{2} \mathrm{MnSn}\left(\mathrm{Co}_{2} \mathrm{FeSi}\right) / \mathrm{AFM}$ bilayers. In $\mathrm{Co}_{2} \mathrm{FeSi} / \mathrm{IrMn}$, a weak EB with $H_{E B}=10$ Oe was already established even in a disordered as-deposited $\mathrm{Co}_{2} \mathrm{FeSi}$ phase (not shown). Further annealing of $\mathrm{Co}_{2} \mathrm{FeSi} / \mathrm{IrMn}$ resulted in an increase of $H_{E B}$. The highest $H_{E B}$ values were obtained applying IrMn as AFM since, in opposite to NiMn, IrMn does not require additional long-term annealing to establish antiferromagnetic ordering. ${ }^{23}$ Therefore, only the data concerning IrMn as AFM will be further discussed. Insertion of an ultrathin Co layer and multiplication of HA/AFM interfaces resulted in an enhancement of $H_{E B}$ except for the $\mathrm{Ni}_{2} \mathrm{MnSn} / \mathrm{AFM}$ layer systems (compare Figs. 4 and 6).

Our results suggest that exchange bias in HA/AFM layers is more subtle than that in conventional FM/AFM structures and no or a very small EB in the as-deposited structures as well as diverse EB depending on HA are worth commenting. In Mn containing $\mathrm{X}_{2} \mathrm{YZ} \mathrm{HA}$, structural ordering is a prerequisite of their ferromagnetic behavior. ${ }^{5}$ If a $\mathrm{Ni}_{2} \mathrm{MnSn}$ film is deposited at RT, it is nanocrystalline and chemically disordered with no sign of ferromagnetism since magnetism in this alloy is extremely sensitive to the distance between the $\mathrm{Mn}$ atoms. ${ }^{4}$ In opposite, $\mathrm{Co}_{2} \mathrm{FeSi}$ alloy is ferromagnetic even in a disordered A2 structure. ${ }^{14}$ Hence, some exchange bias can be established even in a disordered $\mathrm{Co}_{2} \mathrm{FeSi}$ film in contact with IrMn. Moreover, the magnetic moments in ordered HA are localized on different atomic sites quite differently (Table I). While in $\mathrm{Co}_{2} \mathrm{FeSi}$ substantial magnetic moments are localized both on $\mathrm{Co}$ and $\mathrm{Fe}$ sites, the magnetic moments in $\mathrm{Ni}_{2} \mathrm{MnSn}$ are mainly localized on the Mn sites and exchange coupled through oscillatory interactions (RKKY) via conduction electrons. ${ }^{2} \mathrm{Co}_{2} \mathrm{MnSn}$ represents an intermediate case but with no sign of ferromagnetic order in the nanocrystalline disordered state. Moreover, the exchange interactions between atoms at various sites vary substantially in the ordered HA. The exchange integrals were calculated using an EMTO package. ${ }^{29}$ As it is seen in Table I, the exchange interactions represented by the exchange integrals $J$ are small for $\mathrm{Ni}_{2} \mathrm{MnSn}$ and substantial for $\mathrm{Co}_{2} \mathrm{FeSi}$. $\mathrm{Co}_{2} \mathrm{MnSn}$ represents an intermediate case, again. On mesoscopic scale exchange, integrals of these values lead to the exchange stiffness $A=J S^{2} / a$ ( $a$ is the lattice 
parameter of HA) constants between 0.1 and $3 \times 10^{-6} \mathrm{erg} / \mathrm{cm}$. These values are roughly in the range of those for permalloy $\left(1 \times 10^{-6} \mathrm{erg} / \mathrm{cm}\right)$ and cobalt $\left.\left(2.3 \times 10^{-6} \mathrm{erg} / \mathrm{cm}\right)\right)^{1,30}$

Following a discussion of the $\mathrm{EB}^{31}$ and our elaboration on exchange interatomic interactions in HA (Sec. I), we present in Fig. 8 a model for EB in HA/AFM bilayers that is determined by the exchange HA-AFM interactions within interface thickness $\xi \approx 1 \mathrm{~nm}$ (about two times of lattice parameter $a$ of HA). We attribute the strength of EB to the strength of the exchange interactions between magnetic moments localized on $\mathrm{Mn}, \mathrm{Co}$ and $\mathrm{Mn}$ or $\mathrm{Co}$ and $\mathrm{Fe}$ atoms in $\mathrm{Ni}_{2} \mathrm{MnSn}, \mathrm{Co}_{2} \mathrm{MnSn}$, and $\mathrm{Co}_{2} \mathrm{FeSi}$, respectively. Interface contains uncompensated AFM moments and a small fraction $\varepsilon$ of uncompensated pinned moments. ${ }^{31}$ In Fig. 8, FM interactions are sketched by "wavy" lines and the local HA-AFM exchange coupling is sketched by small "springs" with the thickness proportional to their strength.

Following Ref. 31, the exchange bias field is microscopically expressed as

$$
H_{E B}=\frac{J\left(\varepsilon S_{A F M}\right) S_{F M}}{\xi a M_{F M}} \frac{1}{t_{F M}}
$$

where a reduction factor $\varepsilon$ is related to a small fraction of the uncompensated moments that are pinned, $J$ is the exchange integral, $M_{F M}$ is the magnetization of a FM, $t_{F M}$ its thickness, and $S_{A F M}$ and $S_{F M}$ are the spin values of AFM and FM, respectively. For an ideal interface with the fully pinned AFM moments, $\varepsilon=1$. For typical FM/AFM structures, number of pinned spins is of order of $0.01 .^{31}$ On a mesoscopic scale, $H_{E B}$ can be written as

$$
H_{E B}=\frac{\langle A\rangle}{\xi M_{F M}} \frac{1}{t_{F M}}
$$

since $\langle A\rangle=J\left(\varepsilon S_{A F M}\right) S_{F M} / a$. $H_{E B}$ is expressed by a volume averaged exchange stiffness exchange stiffness $\langle A\rangle=\varepsilon A$ $(\mathrm{erg} / \mathrm{cm})$ across the interfacial region of the thickness $\xi$ instead of an area energy density. Hence, we postulate that $\langle A\rangle \propto A$, which is specific for a given $\mathrm{HA}$ and $\langle A\rangle \propto \varepsilon$, which is mainly determined by interface microstructure (i.e., its roughness, grain sizes of AFM and FM). Taking $\xi=1 \mathrm{~nm}, A_{F M}$ from Table I, $t_{F M}=10 \mathrm{~nm}$, the reasonable values of $\varepsilon=0.001$ and 0.01 , and appropriate HA magnetization $M_{F M}$ of 1000,800 , and $400 \mathrm{G}$ at RT, we find $H_{E B}$ of

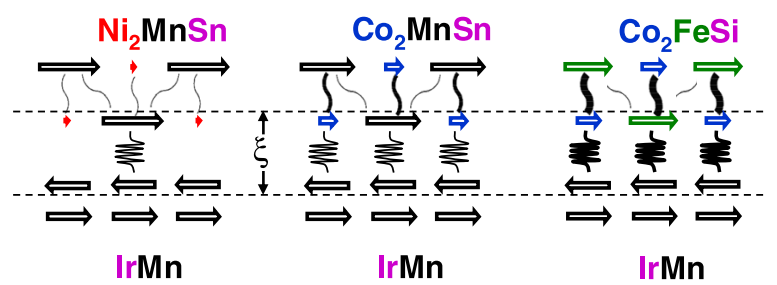

FIG. 8. Schematic illustration of the distribution of the magnetic moments and the magnetic couplings in $\mathrm{Ni}_{2} \mathrm{MnSn}, \mathrm{Co}_{2} \mathrm{MnSn}$, and $\mathrm{Co}_{2} \mathrm{FeSi}$ Heusler alloy films in contact with IrMn. The black, blue, green, and red arrows depict magnetic moments on $\mathrm{Mn}, \mathrm{Co}, \mathrm{Fe}$, and $\mathrm{Ni}$, respectively. The magnitude of the moments is represented by their length. Wavy lines and small "springs" depict the interatomic exchange between atomic moments in these alloys and local FM/AFM exchange coupling, respectively. Their thickness is roughly proportional to the strength of interactions.
$30,7.5,2.5 \mathrm{Oe}$ and 300,75 , and $25 \mathrm{Oe}$ for $\mathrm{Co}_{2} \mathrm{FeSi}$, $\mathrm{Co}_{2} \mathrm{MnSn}$, and $\mathrm{Ni}_{2} \mathrm{MnSn}$ bilayers and multilayers, respectively, in a rough agreement with the experimental data. Therefore, $H_{E B}$ should be approximately the same for the structures deposited and annealed at the same conditions and different for bilayers and for multilayers.

In Fig. 9, we plot a dependence of $H_{E B}$ on $A$ both for HA/ IrMn bilayers and multilayers. Despite of scattered data points, the linear increase of $H_{E B}$ with $A$ predicted by Eq. (2) is supported by the experimental data with various slopes for bilayers, multilayers, and Co dusted HA/IrMn multilayers, respectively. A higher slope of $H_{E B}$ vs. $A$ observed for multilayers in comparison with that of bilayers is attributed to a better microstructure (grain size and the preferred (111) texture of IrMn grains) for the upper stacks as well as doubled FM/ AFM interfaces. Both may lead to a higher $\varepsilon$, i.e., the increase in percentage of the pinned moments. On the grounds of our experience of $\mathrm{Co} / \mathrm{Au}$ multilayers ${ }^{32}$ and the observed increase of the mean grain size after annealing, we attribute the "fine" structure of EB shown in Figs. 6(c) and 6(d) to increasing grain sizes from the bottom stack to top stack. We did not investigate systematically grain-size matching between HA and AFM layers except a rough estimation of grain-sizes using the Scherrer formula for the XRD data. The results of other investigations suggest that the grain sizes in the top stack should be even larger than $11-12 \mathrm{~nm}^{15}$ Doubled FM/ AFM interfaces in multilayers may have some positive effect on enhancement of EB though they can be seen as the multiplied top-and bottom-pinned structures. Usually, in FM/AFM bilayers with a top-pinned AFM uncompensated moments in AFM are set in during deposition. This gives rise to some exchange bias already in the as-deposited state. In the bottompinned AFM/FM bilayers, the exchange bias is often much

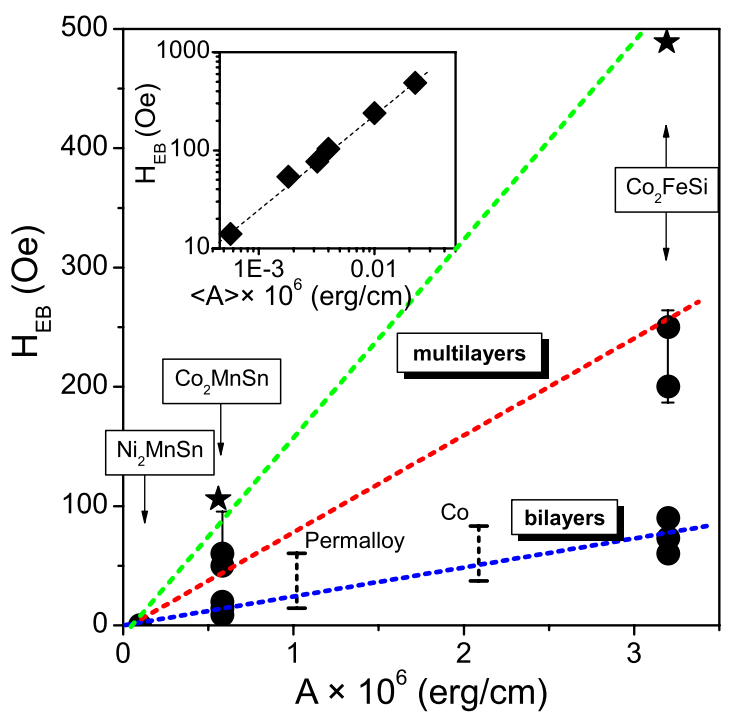

FIG. 9. Dependence of $H_{E B}$ on exchange stiffness A of bilayers and multilayers of $10 \mathrm{~nm} \mathrm{Co} \mathrm{Co}_{2} \mathrm{FeSi}, \mathrm{Co}_{2} \mathrm{MnSn}$, and $\mathrm{Ni}_{2} \mathrm{MnSn} \mathrm{HA}$ in contact with 10-20 nm thick IrMn. For comparison, the dashed bars denote $H_{E B}$ values of permalloy/IrMn (Ref. 34) and Co/IrMn (Ref. 35) bilayers of approximately the same thickness. Asterisks denote the highest values of $H_{E B}$ for $\mathrm{Co}_{2} \mathrm{MnSn} / \mathrm{IrMn}$ and $\mathrm{Co}_{2} \mathrm{FeSi} / \mathrm{IrMn}$ multilayers with ultrathin Co dusted at interfaces. Inset shows $H_{E B}$ vs. $\langle A\rangle$ in log-log scale using the reduction factors $\varepsilon=0.001,0.003$, and 0.007 for bilayers, multilayers, and Co dusted multilayers, respectively. 
smaller since the internal spin structure is developed in the AFM layer before deposition of the FM layer. We confirm the similar differences between the bottom-pinned $\operatorname{IrMn}(20) /$ $\mathrm{Co}_{2} \mathrm{FeSi}(10)$ and the top-pinned $\mathrm{Co}_{2} \mathrm{FeSi}(10) / \mathrm{IrMn}(20)$ bilayer with $H_{E B}=0 \mathrm{Oe}$ and $5 \mathrm{Oe}$ in the as-deposited state, respectively. After annealing at 400 for $15 \mathrm{~min}$ and field cooling, the bottom-pinned bilayer had $H_{C}=112 \mathrm{Oe}$ and $H_{E B}=15 \mathrm{Oe}$ while the top-pinned bilayer had $H_{C}=230 \mathrm{Oe}$ and $H_{E B}=50$ Oe. Therefore, $\mathrm{EB}$ in the $\mathrm{Co}_{2} \mathrm{FeSi} / \mathrm{IrMn}$ multilayers is probably determined by the top-pinned IrMn in each stack. An increase in $H_{E B}$ due to "dusting" with Co can be attributed to a further increase in $\langle A\rangle$ within the interface region $\xi$. We estimate a ratio of the slopes in Fig. 9 to be about 1:3.3:7 for the bilayers, multilayers, and Co dusted multilayers, respectively. Taking somewhat arbitrary $\varepsilon \approx 0.001$ for bilayers and the ratio of slopes in Fig. 9, we plot in the inset of Fig. $9 H_{E B}$ vs. $\langle A\rangle$ for all data points in Fig. 9 to show that the linear trend is preserved in a wide range of $\langle A\rangle$ in agreement with Eq. (2).

\section{CONCLUSIONS}

We showed that the origin of exchange bias in Heusler alloy films in contact with an antiferromagnet is more complex than that for conventional FM/AFM structures. We qualitatively explain the strength of EB in HA/AFM structures as depending on magnitude of exchange integrals between magnetic moments localized in HA in a different way. On mesoscopic scale, the exchange interactions are described by the exchange stiffness constant $A$. Our results show that $\mathrm{EB}$ in $\mathrm{HA} / \mathrm{IrMn}$ structures is proportional to $A$ scaled by a reduction factor $\varepsilon \approx 0.001-0.01$, which is ascribed to a small number of the pinned spins. Our experimental results of EB in bilayers and multilayers as well as Co "dusted" multilayers suggest that interface roughness and crystallite sizes determine the magnitude of the reduction factor $\varepsilon$. In Mn containing HA/AFM structures, a strong increase in EB is observed at low temperatures. We preferably relate this effect to FM/AFM interactions in nanoscale rather than to HA/IrMn coupling at interfaces.

\section{ACKNOWLEDGMENTS}

We thank Dr. B. Szymański, Mr. M. Matczak, and Mr. A. Krysztofik for their assistance with measurements. Authors acknowledge support from Polish Ministry of Higher Education and Science (Grant No. 733/N-DAAD/ 2010/0) for financial support. K.Z. would like to thank to I. A. Abrikosov, B. Alling, and M. Ekholm from Linköping University for their assistance with calculations using the EMTO package.
${ }^{1}$ J. Nogués, J. Sort, V. Langlais, V. Skumryev, S. Suriñach, J. S. Muñoz, and M. D. Baró, Phys. Rep. 422, 65 (2005).

${ }^{2}$ P. J. Webster, J. Phys. Chem. 32, 1221 (1971).

${ }^{3}$ J. Kübler, A. R. Williams, and C. B. Sommers, Phys. Rev. B 28, 1745 (1983).

${ }^{4}$ T. Graf, C. Felser, and S. S. P. Parkin, Prog. Solid State Chem. 39, 1 (2011).

${ }^{5}$ J. Dubowik, I. Gościańska, A. Szlaferek, and Y. V. Kudryavtsev, Mater. Sci. (Poland) 25, 583 (2007).

${ }^{6} \mathrm{~S}$. Kämmerer, "The Heusler alloy $\mathrm{Co}_{2} \mathrm{MnSi}$ in thin films," Ph.D. dissertation (Bielefeld University, 2004).

${ }^{7}$ T. Ishikawa, H.-x. Liu, T. Taira, K.-i. Matsuda, T. Uemura, and M. Yamamoto, Appl. Phys. Lett. 95, 232512 (2009).

${ }^{8}$ M. Oogane, T. Kubota, Y. Kota, S. Mizukami, H. Naganuma, A. Sakuma, and Y. Ando, Appl. Phys. Lett. 96, 252501 (2010).

${ }^{9}$ J. Dubowik, I. Gościańska, K. Załęski, H. Głowiński, A. Ehresmann, G. Kakazei, and S. A. Bunyaev, Acta Phys. Pol. A 121, 1121 (2012).

${ }^{10}$ Y. Sakuraba, M. Hattori, M. Oogane, H. Kubota, Y. Ando, A. Sakuma, and T. Miyazaki, J. Phys. D: Appl. Phys. 40, 1221 (2007).

${ }^{11}$ Y. Ohdaira, M. Oogane, H. Naganuma, and Y. Ando, Appl. Phys. Lett. 99, 132513 (2011).

${ }^{12}$ W. Wang, H. Sukegawa, and K. Inomata, Phys. Rev. B 82, 092402 (2010).

${ }^{13}$ N. P. Alley, S. Takayama, A. Hirohata, and K. O'Grady, IEEE Trans. Magn. 47, 3490 (2011).

${ }^{14}$ H. Endo, A. Hirohata, T. Nakayama, and K. O'Grady, J. Phys. D: Appl. Phys. 44, 145003 (2011).

${ }^{15}$ H. Endo, A. Hirohata, J. Sagar, L. R. Fleet, T. Nakayama, and K. O’Grady, J. Phys. D: Appl. Phys. 44, 345003 (2011).

${ }^{16}$ SimulReflec, Copyright (C) Lab. Lèon Brillouin CEA/CNRS UMR12. Free software available at www.llb.cea.fr/prism/programs/simulreflec.html.

${ }^{17}$ S. Yamada, K. Mamaya, K. Yamamoto, T. Murakami, K. Mibu, and M. Miyao, Appl. Phys. Lett. 96, 082511 (2010).

${ }^{18} \mathrm{Z}$. Li, Ch. Jing, J. Chen, Sh. Yuan, Sh. Cao, and J. Zhang, Appl. Phys. Lett. 91, 112505 (2007).

${ }^{19}$ I. Gościańska, K. Załęski, H. Głowiński, Y. V. Kudryavtsev, and J. Dubowik, Acta Phys. Pol. A 121, 1179 (2012).

${ }^{20}$ K. Westerholt, U. Geiersbach, and A. Bergmann, J. Magn. Magn. Mater 257, 239 (2003).

${ }^{21}$ H. C. Kandpal, G. H. Fecher, and C. Felser, J. Phys. D: Appl. Phys. 40, 1507 (2007).

${ }^{22}$ S. Trudel, O. Gaier, J. Hamrle, and B. Hillebrands, J. Phys. D: Appl. Phys. 43, 193001 (2010).

${ }^{23}$ T. Lin, D. Mauri, N. Stand, C. Hwang, J. K. Howard, and G. L. Gorman, Appl. Phys. Lett. 65, 1183 (1994).

${ }^{24}$ J. P. Nozieres, S. Jaren, Y. B. Zhang, K. Pentek, A. Zeltser, P. Wills, and V. P. Speriosu, J. Appl. Phys. 87, 3920 (2000).

${ }^{25}$ J. W. Cai, K. Liu, and C. L. Chien, Phys. Rev. B 60, 72 (1999).

${ }^{26}$ K. Takano, R. H. Kodama, and A. E. Berkowitz, Phys. Rev. Lett. 79, 1130 (1997).

${ }^{27}$ L. Vitos, Phys. Rev. B 64, 014107 (2001).

${ }^{28}$ S. K. Bose, J. Kudrnovský, V. Drchal, and I. Turek, Phys. Rev. B 82, 174402 (2010).

${ }^{29}$ Materials Modelling Laboratory, IFM, Linkoping Univ., EMTO code, https://wiki/ifm.liu.se/mml/emto.

${ }^{30}$ J. Hamrle, O. Gaier, S.-G. Min, B. Hillebrands, Y. Sakubara, and Y. Ando, J. Phys. D: Appl. Phys. 42, 084005 (2009).

${ }^{31}$ J. Stöhr and H. C. Siegmann, Magnetism: From Fundamentals to Nanoscale Dynamics (Springer, Berlin, 2006).

${ }^{32}$ F. Stobiecki, private communication (2013).

${ }^{33}$ S. Giri, M. Patra, and S. Majumdar, J. Phys.: Condens. Matter 23, 073201 (2011).

${ }^{34}$ C. Tsang, N. Heiman, and K. Lee, J. Appl. Phys. 52, 2471 (1981).

${ }^{35}$ K. A. Seu, H. Huang, J. F. Lesoine, H. D. Showman, W. F. Egelhoff, L. Gan, and A. C. Reilly, J. Appl. Phys. 93, 6611 (2003). 